\title{
26-Week Repeated Dose Oral Toxicity Study of KCHO-1 in Sprague-Dawley Rats
}

\author{
Muhack Yang ${ }^{1,2 \dagger}$, Seongjin Lee ${ }^{1,2 \dagger}$, Tingting Wang ${ }^{1,2+}$, Eunhye Cha ${ }^{1,2}$, Jongwon Jang ${ }^{1,2}$, \\ Dongwoung Kim ${ }^{3}$, Bong-Keun Song ${ }^{4}$, llhong Son ${ }^{4}$, Joonyup Kim ${ }^{4}$, Hyung Won Kang ${ }^{5}$, \\ Sungchul Kim ${ }^{1,2 *}$
}

\author{
'Department of Acupuncture \& Moxibustion Medicine, Wonkwang University Gwangju Korean Medicine Hospital, Gwangju, 61729, \\ Republic of Korea \\ ${ }^{2}$ Nervous \& Muscular System Disease Clinical Research Center of Wonkwang University Gwangju Korean Medical Hospital, Gwangju, 61729 , \\ Republic of Korea \\ ${ }^{3}$ Department of Internal Medicine, Wonkwang University Gwangju Korean Medicine Hospital, Gwangju, 61729, Republic of Korea \\ ${ }^{4}$ Department of Neurology, InAm Neuroscience Center, Sanbon Hospital, Wonkwang Unversity School of Medicine, Gunpo, 15865, \\ Republic of Korea \\ ${ }^{5}$ Department of Neuropsychiatry of Korean Medicine \& Inam Neuroscience Research Center, Wonkwang University Sanbon Hospital. \\ Gunpo, 15865, Republic of Korea
}

\section{Key Words}

KCHO-1(Mecasin), 26-Week Chronic Oral Toxicity Test, NOAEL(No observed adverse effect level), Sprague-Dawley Rats

\section{Abbreviations}

No observed adverse effect level =NOAEL, Sprague-Dawley $=$ SD, Korea Testing \& Research Institute $=$ KTR, Good Laboratory Practice $=$ GLP, Body Weight $=$ BW .

\section{Abstract \\ Objectives: KCHO-1(Mecasin), also called Gamijakyak- gamchobuja-tang originally, is a combination of some traditional herbal medicines in East Asia. This medicine has been used mainly for alleviating neuropathic pains for centuries in Korean traditional medicine. KCHO-1 was developed to treat pain, joint contracture and mus- cular weakness in patients with amyotrophic lateral sclerosis. This study was carried out to investigate the chronic toxicity of KCHO-1 oral administration in rats}

for 26 weeks.

Methods: Sprague-Dawely rats were divided into four groups and 10 rats were placed in the control group and the high-dose group, respectively. Group 1 was the control group and the remaining groups were the experimental groups. In the oral toxicity study, $500 \mathrm{mg}$ / $\mathrm{kg}, 1,000 \mathrm{mg} / \mathrm{kg}$, and 2,000 mg/kg of KCHO- 1 were administered to the experimental group, and $10 \mathrm{ml} / \mathrm{kg}$ of sterile distilled water was administered to the control group. Survival rate, body weight, feed intake, clinical signs, and visual findings were examined. Urinalysis, ophthalmologic examination, necropsy, organ weight, hematologic examination, blood chemical examination and histopathologic examination were performed.

Results: Mortality and toxicological lesions associated with the administration of test substance were not observed in all groups.

Conclusion: NOAEL(No observed adverse effect level) of KCHO-1 is higher than $2000 \mathrm{mg} / \mathrm{kg} /$ day. And, the above findings suggest that treatment with $\mathrm{KCHO}-1$ is relatively safe.

Received: May 28, 2019 Reviewed: Jun 14, 2019 Accepted: Sep 03, 2019

(c) This is an Open-Access article distributed under the terms of the Creative Common Attribution Non-Commercial License (http://creativecommons.org/licenses/by-nc/4.0/) which permits unrestricted noncommercial use, distribution, and reproduction in any medium, provided the original work is properly cited.

$\otimes$ This paper meets the requirements of KS X ISO 9706, ISO 9706-1994 and ANSI/NISO Z39.48-1992 (Permanence of Paper).
Corresponding Autho

Sungchul Kim. Wonkwang University Gwangju Korean Medical Hospital, 1140-23, Hoejae-ro, Nam-gu, Gwangju, 61729, Korea.

Tel: +82-62-670-6441 Fax: +82-62-670-6767 E-mail: kscndl@hanmail.net $\dagger \mathrm{MY}$, SL and TW equally contributed. (co-first authors)

(c) 2019 Korean Pharmacopuncture Institute

http://www.journal.ac 


\section{Introduction}

KCHO-1(Mecasin) is herbal medicine called as Gamijakyakgamchobuja-tang originally in Korean traditional medicine. This concoction is reported to have anti-inflammatory and antioxidant properties [14]. KCHO-1 was developed for treating amyotrophic lateral sclerosis patients with pain, joint contracture and muscular weakness and has been used in traditional medicine to relieve pain, muscle spasms and cold syndrome due to blood deficiency [5]. In the previous study, it was found that KCHO-1 has a reduced pain, GABA neuron regeneration and nitric oxide reduction in neuropathic pain rats [14]. In addition, Jakyakgamcho-tang consisted of some constituent herbs in KCHO-1, has been studied for anti-seizure, analgesic, antipyretic, anti-inflammatory and anti-ulcer effects [35,13], and its constituents have a protective effect against tert-butyl hydroperoxide (t-BHP)-induced cytotoxicity in the hippocampal HT22 cell line [12]. And Jakyakgamchobuja-tang, herbal formula also consisted of some constituent herbs in KCHO-1, is believed to be useful for suppressing the progress of osteoarthritis because of its anti-inflammatory effects and its ability to reduce pain with histopathological efficacy [22]. Because of these facts, KCHO-1 has been studied for treating patients with joint pain, muscle spasms, and arthralgia due to cold. Several studies have been conducted about concerning the toxicity and the efficacy of these constituent. Seo et al. [29] studied the hypokalemia and myopathy induced by Radix Glycyrrhizae. Kwak et al. [18] conducted a study on the cytotoxicity and antimicrobial effect of extracts from Salvia miltiorrhiza. Kim et al. [16] conducted a study on the single-dose toxicity of Aconitum Kusnezoffii Reichb. pharmacopuncture in rats and suggested that Aconitum kusnezoffii Reichb. pharmacopuncture was relatively safe. Han et al. [6] studied a 2-week repeated oral toxicity of Polygalae Radix Aqueous extract and recommended that a dose group of $3500 \mathrm{mg} / \mathrm{kg} /$ day was a highest treatment group in 13-week exposure study. Kim et al. [17] conducted a philological study on the poisoning and the side effects of Rhizoma Gastrodiae. Choi et al. [2] conducted a study on the cytotoxicity and the antimicrobial effects of extracts from the Atractylodes japonica Koidzumi. And, single dose toxicity test [10] and 4-week repeated oral dose toxicity test of KCHO-1 [1] were performed. Nevertheless, chronic toxicity testing of K-CHO1 has not been conducted yet.

This study was conducted to evaluate the chronic toxicity and confirm the NOAEL (No observed adverse effect level). In this study, oral administration of KCHO-1 to male and female rats was repeated for 26 weeks. All the experiments for this research were conducted at the Korea Testing \& Research Institute (KTR), an institution authorized to perform non-clinical studies, under the Good Laboratory Practice (GLP) regulations, also accredited by AAALAC International (Association for Assessment and Accreditation of Laboratory Animal Care, IAC2016-1491).

\section{Material and Methods}

\section{1. $\mathrm{KCHO}-1$ preparation}

KCHO-1 is consisted of nine herbs, Radix Paeoniae Alba (Paeonia lactiflora PALL.), Radix Glycyrrhizae (Glycyrrhiza uralensis FISCH.), Radix Aconiti Lateralis Preparata (Aconitum carmichaeli DEBX.), Radix Salviae Miltiorrhizae (Salvia miltiorrhiza BGE.), Rhizoma Gastrodiae (Gastrodia elata BL.), Rhizoma Curcumae Longae (Curcuma longa L.), Fructus Chaenomelis (Chaenomeles speciosa NAKAI), Rhizoma Atractylodis Japonicae (Atractylodes japonica KOIDZ. ex KITAM.), Radix Polygalae Tenuifoliae (Polygala tenuifolia WILLD.). KCHO-1 was provided by the Nervous \& Muscular System Disease Clinical Research Center of Wonkwang University Gwang-ju Korean Medical Hospital. The test substance was used as it was without conversion into purification, and the concentrations were $50 \mathrm{mg} / \mathrm{mL}, 100 \mathrm{mg} / \mathrm{mL}$, and $200 \mathrm{mg} / \mathrm{mL}$. The completed extracts were stored at room temperature $\left(1-30^{\circ} \mathrm{C}\right)$.

\subsection{Animal}

\subsubsection{Animals and Husbandry}

The animals used in oral dose toxicity test were 5-week old Sprague-Dawley (SD) rats. The reason SD rats were chosen is that they have been widely used on safety test in the field of medicine and they can be compared with a lot of other data bases easily. The mean weights of the rats were $112.9 \sim 139.3 \mathrm{~g}$ and $93.0 \sim 111.5 \mathrm{~g}$ for the male and the female rats at the time of oral dose toxicity test. For all animals, a visual inspection was conducted.

The temperature of the lab was $20.9 \sim 23.8^{\circ} \mathrm{C}$, and the humidity was $47.6 \sim 62.8 \%$. Enough food (Rodent Diet 20 5053) and UV-filtered water were provided. The temperature and humidity of the animal room were measured every 30 minutes by an automatic temperature and humidity meter. The environmental conditions such as illumination were measured according to standard work instructions. Variation that would affect the test results was not observed.

\subsubsection{Grouping}

After acclimatization, healthy individuals were selected, and grouping was performed randomly so that the mean weight and standard deviation among the groups were equal. (Table 1) After grouping, the remaining animals were anesthetized with isoflurane and euthanized via exsanguination.

\subsubsection{Dosing}

No toxicological changes related to the administration of the test substance were observed in the high-dose of 2000 $\mathrm{mg} / \mathrm{kg} \mathrm{B.W}$. of the 4 -week repeated dose determination test using this test substance. Therefore, the high-dose was set at $2000 \mathrm{mg} / \mathrm{kg} \mathrm{B.W.,} \mathrm{and} \mathrm{the} \mathrm{geometric} \mathrm{ratio} \mathrm{was}$ set at 2, the mid-dose was set at $1000 \mathrm{mg} / \mathrm{kg} \mathrm{B.W.,} \mathrm{and} \mathrm{the}$ low-dose was set at $500 \mathrm{mg} / \mathrm{kg} \mathrm{B.W}$. The control group was administered with the same amount of excipient as the experimental group. In addition, in order to evaluate the reversibility of the toxicity change according to the test substance administration, the recovery group was set separately in the control group and the high- dose group. 
During the administration of the test substance preparation, it was directly administered into the stomach using a syringe with a sonde for administration while shaking by hand. The test substance was administered to the SD rats 7 days/week, 1 time/day for 26 week. And the dose (mL/ $\mathrm{kg}$ ) was calculated based on the most recently measured body weight at the time of administration.

Table 1 Groups of Animals

\begin{tabular}{|c|c|c|c|c|c|c|}
\hline \multirow[b]{2}{*}{$\begin{array}{l}\text { Grou } \\
\mathrm{p}\end{array}$} & \multirow[b]{2}{*}{ Sex } & \multirow[b]{2}{*}{$\begin{array}{l}\text { Serial } \\
\text { number }\end{array}$} & \multicolumn{2}{|c|}{ Number of animals } & \multirow{2}{*}{$\begin{array}{l}\text { K-CHO1 } \\
\text { administration } \\
\text { (mg/kg B.W.) }\end{array}$} & \multirow{2}{*}{$\begin{array}{l}\text { Oral } \\
\text { dose } \\
\text { (mL/kg } \\
\text { B.W.) }\end{array}$} \\
\hline & & & $\begin{array}{l}\text { Administratio } \\
\mathrm{n} \text { group }\end{array}$ & $\begin{array}{l}\text { Recovery } \\
\text { group }\end{array}$ & & \\
\hline \multirow{2}{*}{ G1 } & Male & $\begin{array}{l}1101- \\
1115\end{array}$ & 10 & 5 & \multirow{2}{*}{0} & \multirow{2}{*}{10} \\
\hline & $\begin{array}{l}\text { Fema } \\
\text { le }\end{array}$ & $\begin{array}{l}2101- \\
2115\end{array}$ & 10 & 5 & & \\
\hline G2 & $\begin{array}{l}\text { Fema } \\
\text { le }\end{array}$ & $\begin{array}{l}\text { 1201- } \\
1210 \\
2201- \\
2210\end{array}$ & 10 & - & 500 & 10 \\
\hline G3 & $\begin{array}{l}\text { Fema } \\
\text { le }\end{array}$ & $\begin{array}{l}\text { 1301- } \\
1310 \\
2301- \\
2310\end{array}$ & 10 & - & 1000 & 10 \\
\hline G4 & $\begin{array}{l}\text { Fema } \\
\text { le }\end{array}$ & $\begin{array}{l}1401- \\
1415 \\
2401- \\
2415\end{array}$ & 10 & 5 & 2000 & 10 \\
\hline
\end{tabular}

\subsection{Observations}

\subsubsection{General symptoms}

During the experimental period, all animals were observed for changes in general symptoms, toxic symptoms, and deaths when before and after the administration during administration period and once a day during the recovery period.

\subsubsection{Weight measurements}

Body weight was measured once a week at the time of introduction, separation of group, and up to 3 months after initiation of administration. After that, it was measured once every 4 weeks and once a week during the recovery period. In order to calculate the relative organ weights (\%), fasting weight was measured on the day of the necropsy and measured at the time of death or moribund an- imal observation.

\subsubsection{Feed intakes}

Feed intake was measured once a week before the start of administration, up to three months after initiation of administration. After that, it was measured once every 4 weeks and once a week during the recovery period. The average daily intake (g/rat/day) was calculated by dividing the difference of the amount of daily dose and the remaining amount of the day after feeding into numbers per cage. The average daily intake (g/rat/day) was calculated by dividing the difference between the amount of daily dose and remaining amount of the next day after feeding into number of per cage. In the case of before administration, the daily intake from the day of grouping to the day of administration was measured.

\subsubsection{Urinalysis}

For each group, 5 male and 5 female animals were examined for the following items in the final week of administration period and the final week of recovery period. Urine was subjected to urine chemistry test, urine sedimentation and urine color test for fresh urine obtained using metabolic cage. And urinary volume stored for about 24 hours was measured.

\subsubsection{Ophthalmological examination}

For all animals in the high- dose and control groups, the appearance of the animal eyes was made an ocular inspection before administration, at the final week of the administration period, and at the final week of the recovery period. After the ocular inspection, the pupil was dilated using a mydriatica [1 \% Mydriacyl (Tropicamide 1\%), Alcon, Belgium], and an ophthalmological examination was performed using an ophthalmoscope (Genesis, Kowa, Japan).

\subsubsection{Necropsy}

During the planned necropsy, surviving animals were anesthetized with isoflurane and exsanguinated and euthanized. Then, the external surface \& all orifices, cranial cavity, thoracic and abdominal cavities \& their contents were conducted macroscopy. The animals that died during the administration were also conducted macroscopy about the external surface \& all orifices, cranial cavity, thoracic and abdominal cavities \& their contents.

\subsubsection{Organ weights}

At the time of necropsy, the wet weight of the following organ was measured for all animals, and the relative organ weight ratio to the fasting weight was calculated: brain, pituitary, heart, lung, liver, spleen, kidneys, adrenals, tested, epididymides, ovaries, uterus, thymus, prostate, salivary gland. The bilateral organs were measured separately and the weights were summed up and calculated. 


\subsubsection{Hematologic examination}

All animals were anesthetized with isoflurane and exsanguinated from the abdominal artery after fasting for more than 18 hours before the blood collection. $2 \mathrm{~mL}$ of the exsanguinated blood was placed in a CBC bottle (EDTA $2 \mathrm{~K}$, $B D$ vacutainer) containing EDTA and the following items were measured using a Blood analysis apparatus (ADVIA 2120i, Siemens, Germany). Blood clotting time was measured by blood coagulation analyzer (ACL 7000, Instrumentation Laboratory, U.S.A.) using plasma obtained by refrigerated centrifugation $\left(3000 \mathrm{rpm}, 4^{\circ} \mathrm{C} 10\right.$ minutes) in a vacutainer (9NC Sodium citrate, $\mathrm{BD}$ vacutainer).

\subsubsection{Blood chemical examination}

The remaining blood, except the blood used for the hematologic examination and the blood coagulation examination, was coagulated at room temperature and then subjected to refrigerated centrifugation $\left(3000 \mathrm{rpm}, 4^{\circ} \mathrm{C}\right.$, 10minutes). The obtained serum was measured using a biochemical analyzer (TBA-120FR, TOSHIBA, Japan) as follows.

\subsubsection{Histopathologic examination}

The following organs were isolated from each animal and fixed in $10 \%$ neutral buffered formalin: liver, kidneys, adrenals, pituitary gland, spleen, brain, heart, Lung and bronchus, testes, seminal vesicle, ovaries, epididymides, uterus, prostate gland, vagina, tongue, trachea, esophagus, thymus, thyroid gland, stomach, parathyroid gland, urinary bladder, small/large intestine, eyeball, submandibular gland, pancreas, sciatic nerve, sternum, mammary gland, femur, spinal cord, skin, mesenteric lymph node. Among them, the testes (epididymis) were fixed in Bouin fixative and the eye was fixed in Davidson solution. Among fixed organs tissues, all organs of control and high-dose groups were subjected to general tissue processing such as dehydration, paraffin infiltration, tissue paraffin embedding, and dissection. And sample slides were fabricated and Hematoxylin \& Eosin (H \& E) staining was performed. After sample fabrication, the remaining and fixed organ $\bullet$ tissues were stored in $10 \%$ neutral buffered formalin.

\subsection{Statistical Analysis}

The weight, feed intake, hematologic examination, Blood chemical examination and organ weight data from the experiments were analyzed by using SPSS program (Ver. 19.0). A Levene test was conducted to evaluate the homogeneity of the variance. The one-way ANOVA test was conducted to confirm the significance of the variance. One way ANOVA test was carried out and the significance between experimental groups was confirmed.

Therefore, the post-hoc test (Scheffe multiple test if variance is homogenous, Dunnett`s T3 if variance is heterogeneous) was conducted according to the presence or absence of homogeneity of variance. In the recovery group, statistical significance test between experimental groups was performed by the independent sample T- test.

\section{Results}

\subsection{Mortalities and general symptoms}

One rat died in the male high-dose group during the experimental period. Except for that, there were no deaths or moribund rats occurred in any of the groups. As a result of observing general symptoms, no animals showed any unusual changes in all groups. (Table 2)

Table 2 Mortality of animals

\begin{tabular}{|c|c|c|c|}
\hline \multirow{2}{*}{$\begin{array}{l}\text { Group } \\
\text { /Dose }(\mathrm{mg} / \mathrm{kg})\end{array}$} & \multirow{2}{*}{ No. of animals } & \multicolumn{2}{|c|}{ Mortality(dead/total) } \\
\hline & & Male & Female \\
\hline G1 & 15 & $0 \%$ & $0 \%$ \\
\hline 0 & $\left(5^{\mathrm{a}}\right)$ & $(0 / 15)$ & $(0 / 15)$ \\
\hline$\overline{\mathrm{G} 2}$ & & $0 \%$ & $0 \%$ \\
\hline 500 & 10 & $(0 / 15)$ & $(0 / 15)$ \\
\hline G3 & & $0 \%$ & $0 \%$ \\
\hline 1000 & 10 & $(0 / 15)$ & $(0 / 15)$ \\
\hline G4 & 15 & $7 \%$ & $0 \%$ \\
\hline 2000 & $\left(5^{\mathrm{a}}\right)$ & $(1 / 15)$ & $(0 / 15)$ \\
\hline
\end{tabular}

a: recovery group

\subsection{Weight changes}

In this study, there were no statistically significant changes between the control groups and the experimental groups.

\subsection{Feed intakes}

Feed intakes were significantly decreased $(\mathrm{P}<0.05)$ in the 2, 3, 4, 5, 11, 21, 25-week low-dose and 25-week highdose groups than in the control group in male and 2-week female high dose group were significantly increased $(\mathrm{P}<$ $0.05)$. In recovery group, no significant difference was observed compared with the control group.

\subsection{Urinalysis}

As a result of urinalysis, no animals showed any unusual changes in all groups.

\subsection{Ophthalmological examination}

As a result of ophthalmological examination, no abnormalities occurred in all groups. 


\subsection{Necropsy}

In both control and experimental groups, no meaningful changes in the necropsy were noted.

\subsection{Organ weights}

As As a result of absolute organ weight measurement, salivary gland and adrenal gland were significantly increased $(\mathrm{P}<0.05)$ in male recovery group compared to control group. As a result of comparative organ weight measurement, epididymis was significantly decreased $(\mathrm{P}<0.05)$ in male recovery group compared to control group. There were no significant differences in absolute and comparative organ weight of male and female experimental groups and female recovery group compared to the control group.

\subsection{Hematologic examination}

Hematologic examination results showed no significant difference in all experimental groups compared to the control group.

\subsection{Blood chemical examination}

Blood chemical examinations showed that the Potassium in the male high-dose group was significantly increased ( $\mathrm{P}$ $<0.05$ ) and the Sodium in the male mid-dose group was decreased $(\mathrm{P}<0.05)$ compared to the control group. In the recovery group, the Aspartate aminotransferase, Glucose, Potassium were significantly decreased $(\mathrm{P}<0.05)$ compared to the control group. In females, no significant difference was observed between the experimental and recovery groups.

\subsection{Histopathologic examination}

Histopathologic examination revealed no lesions associated with the administration of the test substance in the organs of the high-dose groups of male and female. As a background lesion, inflammatory cell infiltration of the liver, denaturation and regeneration of the renal tubule, adrenal cortical vacuolation, interstitial inflammatory cell infiltration of prostate gland were observed in the male high-dose group, and inflammatory cell infiltration of the liver, denaturation and regeneration of the renal tubule, kidney medullary mineralization were observed in the female high-dose group. Inflammatory cell infiltration of the liver was observed in the male high-dose recovery group, and inflammatory cell infiltration of the liver, denaturation and regeneration of the renal tubule and regional vasodilation in the adrenal gland were observed female high-dose recovery group. As a contingency lesion, nephropathy was observed in 2 out of 10 male high-dose groups.

\section{Discussion}

KCHO-1 has been utilized in clinics to relieve pain, joint contracture, muscular atrophy and muscular weakness, especially for patients with amyotrophic lateral sclerosis. KCHO-1(Mecasin) is a new medicine based on Jakyagamchobuja-tang from Shang Han Lun(傷寒論) and Jin Kui Yao Lue(金嘈要略). 6 herbal medicines are newly added based on Korean Medicine theory of Gunsinjwasa(君臣 佐使). Its main constituents, Radix paeoniae ALBA, Radix glycyrrhizae and Radix aconite lateralis preparata, have been reported to be effective for osteoarthritis [22]. The other constituents of this herbal formula are Curcuma Root, Rhizoma Gastrodiae, Radix Salviae Miltiorrhizae, Fructus Chaenomelis, Rhizoma Atractylodis Japonicae and Radix Polygalae, which are known to have anti-inflammatory and anti-oxidative activities [14].

Radix Paeoniae ALBA is a herbal medicine that has been used for hundreds of years to treat gastrointestinal disorder [4]. Park and Sohn [27] reported that Radix Paeoniae ALBA extracts pharmacopuncture has anti-inflammatory effects. This pharmacopuncture restrained inflammatory reaction and reduced the number of WBC significantly, also affected Globulin and Albumin in the blood serum. Moreover, muscular tissue necrosis in Freund`s complete adjuvant arthritis of SD rats was restrained. And Huang et al. [8] conducted the study that providing in-vitro and in-vivo evaluations of the use of Radix Paeoniae ALBA for peripheral nerve regeneration. They suggested that Radix Paeoniae ALBA extracts could be a potential nerve growth-promoting factor, being effective on helping the growth of injured peripheral nerves.

Radix Glycyrrhizae has been commonly used in the Orient for treating a variety of diseases. Park et al. [26] reported that administration of Radix Glycyrrhizae improved spatial learning and memory and reduced stress-induced anxiety. They concluded that Radix Glycyrrhizae had the potential to attenuate the behavioral and neurochemical impairments caused by stress. And, Radix Glycyrrhizae extract could be beneficial for inhibition of allergic inflammatory response by inhibiting secretion of $\beta$-hexosaminidase and formation of IL-4 TNF- $\alpha$ [20].

Radix Aconiti lateralis preparata has been widely used for thousands of years to improve symptoms of heart failure, inflammation, pain, and diarrhea. However, the toxicity of Aconiti lateralis preparata Radix has been the subject of disputation [33]. Some kinds of diester diterpenoid-type aconitum alkaloids, such as aconitine and mesaconitine, are major toxic ingredients [32]. However, The herbal acupuncture with Radix Aconiti lateralis preparata can accelerate movement recovery in patients with spinal cord injuries at an early stage [19]. Furthermore, Radix Aconiti lateralis preparata herbal acupuncture has pain-relieving and anti-inflammatory effects on the arthritis induced by Freund's complete Adjuvant in rats. Jeong et al. [11] measured the levels of plasma serotonin, WBC, RBC, $\mathrm{Hb}$, ESR after injecting Aconiti Lateralis Preparta Radix herbal acupuncture. In this experiment, the levels of plasma serotonin, WBC, RBC, $\mathrm{Hb}$, and ESR were decreased and the paw edema of rats was also reduced.

Radix Salviae Miltiorrhizae is one of the most widely used medicinal herbs for gynecological disease, and its efficacy is to eliminate extravasated blood. In clinics, it is used not only for gynecological diseases but also for cardiovascular diseases [15] The antioxidative efficacy of Radix Salviae Miltiorrhizae was studied by Yang et al. [34]. In addition, 
Lee and Kim [21] conducted a study of the effects of Radix Salviae Miltiorrhizae on neuronal apoptosis following intracerebral hemorrhage in rats. They showed that Radix Salviae Miltiorrhizae reduced neuronal apoptosis.

Rhizoma Gastrodiae has been used in the treatment of symptoms such as headache, dizziness, paralysis, and convulsion [23]. Recently, studies about Rhizoma Gastrodiae on the effects of $\gamma$-aminobutyric acid(GABA) neurotransmission regulation, regional cerebral blood flow increase, blood pressure lowering, triglyceride and cholesterol lowering, and neuronal cell protection have been actively conducted [3].

Radix Polygalae is used to treat anxiety, insomnia, forgetfulness [25]. Shin et al. [31] reported that Radix Polygalae extract could protect against $\mathrm{N}$-methyl D-aspartate (NMDA) neurotoxicity and induced brain-derived neurotrophic factor expression, suggesting modulatory roles at glutamatergic synapses and possible antidepressant action.

Curcuma Root has been used to relieve pain including menstrual pain and flank pain in oriental medicine [24]. Quiles et al. [28] reported inhibition effects of Curcuma Root on cell death induced by hydrogen peroxide in SK-NMC neuroblastoma cells.

Fructus Chaenomelis herbal acupuncture at the ST36 has an important role to control the immune reactions and suppress inflammatory response in the collagen-induced rheumatoid arthritis [30].

Recently, the pharmacological analysis of Rhizoma atractylodis japonicae has revealed the anti-inflammatory, analgesic, antipyretic and immune enhancement effects and has been applied to various inflammatory diseases [7]. Furthermore, Hur et al. [9] investigated the effects of Rhizoma atractylodis japonicae against LPS-induced inflammation in mouse microglia BV2 cells, and its extracts were shown to suppress NO production by inhibition of iNOS expression and COX-2 activity.

Thus, KCHO- 1 and its component herbs have been reported to have numerous positive effects on many disorders. Although it has been used in clinics to treat various diseases in these days, safety studies on KCHO-1 are insufficient. Toxicity tests provide important data and are essential for assessing the safety of test substances in medications. All experiments for this research were performed at KTR, an institution authorized to conduct non-clinical studies, under the GLP regulations. Animal testing is the most fundamental and basic method to perform safety assessments.

One rat died in the male high-dose group during the toxicity test period. However, no toxicological changes were observed in the results of general symptoms and histopathological observation until death, and autopsy. Therefore, it is not regarded as death due to test substance administration.

Feed intakes were significantly decreased in the 2, 3, 4, 5, 11, 21, 25-week low-dose and 25-week high-dose groups than in the control group in male and 2-week female high dose group were significantly increased. This is a temporary or not dose-dependent, so is not considered as toxic effects by administration of the test substance. As a result of absolute organ weight measurement, salivary gland and adrenal gland were significantly increased in male recovery group compared to control group. As a result of comparative organ weight measurement, epididymis was significantly decreased in male recovery group compared to control group. However, it is not considered toxic effects by the administration of the test substance, since any sign of abnormalities in general symptoms and pathological examination of the organ was observed. Blood chemical examinations showed that the Potassium in the male high-dose group was significantly increased and the Sodium in the male mid-dose group was decreased compared to the control group. In the recovery group, the Aspartate aminotransferase,

Glucose, Potassium were significantly decreased compared to the control group. However, it is not considered toxicity of the administered test substance because no abnormality of the organs related to the above symptoms was observed. As a result of histopathologic examination, inflammatory cell infiltration of the liver of the male and female high-dose group and the high-dose recovery group was mild, and was also observed in the control group. Therefore, it is considered to be a naturally observable background lesion. Denaturation and regeneration of the renal tubule and medullary mineralization, adrenal cortical vacuolation and regional vasodilation, interstitial inflammatory cell infiltration of prostate gland are background lesions observable in the normal repeated dose toxicity studies, and prevalence and grade of lesions was slight. Moreover, most of the findings were also observed in the control group. So, these symptoms were considered to be background lesions not associated with the administration of the test substance. The nephropathy observed in 2 out of 10 male high-dose groups is called protein-overload nephropathy or chronic progressive nephrosis, and is known to be a degenerative lesion in an old-aged rat. This lesion is difficult to be observed with the naked eye until the lesion becomes severe. In the early stage of the lesion, degeneration and regeneration of the tubule begin concurrently and the lesion progresses to chronic progressive nephropathy accompanied by thickening of the membrane, enlargement of the tubule, fibrosis of the cast in tubule and the epilepsy and infiltration of inflammatory cells. The lesions observed in this study were early-stage nephropathy, low prevalence of lesions, and also observed in one male control recovery group. Considering these factors, it was considered to be a spontaneous lesion, not an effect of test substance administration. Histopathological examination of the dead rat during the examination revealed that most organs were autolysis and toxicological lesions associated with the administration of the test substance were not observed. Histopathological examination of other rats in the high-dose group, which is the same group as the dead rat, did not reveal any lesions associated with the administration of the test substance. So, it is considered that the cause of death of the rats is not the effect of the test substance administration.

The results of our study showed that administration of $2,000 \mathrm{mg} / \mathrm{kg}$ of $\mathrm{KCHO}-1$ did not cause any changes in the weights of SD rats or in the results of necropsy examinations. It indicates that $\mathrm{KCHO}-1$ administration can be used as a safe treatment. The present study was designed to evaluate a 26 -week, repeated, oral dose, toxicity test of KCHO-1 in SD rats. Our study showed no significant chang- 
es in the weight, feed intakes, urinalysis, ophthalmology, hematology, serum biochemistry, organ weights, necropsy findings, and histopathology of the rats. Although some changes were observed in several organs, those changes seemed to have occurred naturally or sporadically. During this 26-week, repeated, oral dose, toxicity test of K-CHO1 in SD rats, no toxicity changes due to KCHO-1 were observed in any of the male or female rats in the high dosage group. In conclusion, the results showed that administration of $500-2,000 \mathrm{mg} / \mathrm{kg}$ BW of KCHO-1 to SD male and female rats did not cause any changes in weight or in the results of necropsy examinations. Although one rat died in the male high-dose group during the toxicity test period, no toxicological changes were observed in the results of general symptoms and autopsy and histopathological observation until death, and therefore, it is not regarded as death due to test substance administration. It indicated that the lethal dose of KCHO-1 was higher than 2,000 $\mathrm{mg} / \mathrm{kg}$. During this 26 -week, repeated, oral toxicity test of KCHO-1 in SD rats, no toxicity changes due to KCHO1 were observed in any of the male or the female rats in the high dosage group. The results obtained in this study suggest that KCHO-1 administration can be used as a safe treatment.

\section{Acknowledgment}

This work was supported by the Korea Health Industry Development Institute [HI11C2142].

\section{Conflict of interest}

The authors declare that there are no conflicts of interest.

\section{Authors contribution}

Writing - original draft and data curation:M. Yang, S. Lee and T. Wang; Validation:E. Cha and J. Jang; Supervision: D. Kim and B. Song; Writing -review \& editing: I. Son, J. Kim, H. Kang; Methodology: S. Kim.

\section{References}

1. Cha EH, Lee JC, Lee SJ, Park MY, Song IJ, Son IH, et al. A 4-week repeated dose oral toxicity study of Mecasin in Sprague-dawley rats to determine the appropriate doses for a 13-week, repeated toxicity test. Journal of Pharmacopuncture. 2015;18(4):45-50.

2. Choi EY, Oh HJ, Park NK, Chun HJ, Ahn JW, Jeon BH, et al. Screening of Cytotoxicity and Antimicrobial Effects of Extracts from the Atractylodes macrocephala Koidz. The Journal of Jeahan Oriental Medical Academy. 2002;16: 348-352.

3. Chu HN, Kim JS, Kim KO, Jeong JK.Effect of functional components, antioxidant activity and sensory characteristics of Gastrodiae Rhizoma by different drying condition. The Korea Journal of Herbology. 2012;27:139145.

4. Fang YS, Shan DM, Liu JW, Xu W, Li CL, Wu HZ, et al. Effect of constituents from Fructus Aurantii Immaturus and Radix Paeoniae Alba on gastrointestinal movement. Planta. Med. 2009;75: 24-31.

5. Guo L, Cho SY, Kang SS, Lee SH, Baek HY, Kim YS. Orthogonal array design for optimizing extraction efficiency of active constituents from Jakyak-Gamcho Decoction, the complex formula of herbal medicines, Paeoniae Radix and Glycyrrhizae Radix. J Ethnopharmacol. 2007;113:306-311.

6. Han HY, Kim SN, Yang YS, Han SC, Seok JH, Roh HS, et al. Toxicity assessment of Polygalae Radix Aqueous extract orally administered to rats for 2 cosecutive weeks. Korean J. Pharmacogn. 2015;46: 44-51

7. Han YK, Park YH, Keum DH, Lee MJ. Effects of Gamichangbaek-san(Jiaweichangbai-san) on Anti-inflammatory, Analgesic, Anti-febrile Activities and Immune Responses in Carrageenan-induced Arthritic Animals. Journal of Oriental Rehabilitation Medicine. 2005;15: 127-141.

8. Huang KS, Lin JG, Lee HC, Tsai FJ, Bau DT, Huang CY, et al. Paeoniae alba Radix Promotes Peripheral Nerve Regeneration. Evid. Based Complement. Alternat. Med. 2011;doi:109809. http://dx.doi.org/10.1093/ecam/ nep115.

9. Hur IH, Shim SY, Kim KJ. Anti-inflammatory effect of various solvent extract from Atractylodes japonica on Lipopolysaccharide-induced Inflammation in BV2 cells. Journal of Korean Medicine Ophthalmology \& Otolaryngology \& Dermatology. 2007;20:36-46.

10. Jeong HH, Lee JC, Cha EH, Park MY, Son IH, Song BK, et al. A study on the oral toxicity of Mecasin in rats. Journal of Pharmacopuncture.2014;17(4): 61-5.

11. Jeong SH, Park DS. The analgesic and anti-inflammatory effect of A.I.P.R aqua-acupuncture on the arthritis induced by Freund's complete Adjuvant in rats. The Acupuncture. 1997;14:334-46.

12. Kang TH, Baek HY, Kim YC. Protective effect of jakyak-gamcho-tang extract and its constituents against t-BHP-induced oxidative damage in HT22 cells. Am. J Chin. Med. 2005;33:181-9.

13. Kim BW. Anti-inflammatory Effect of Jakyakgamcho-tang. Korean J. Orient. Int. Med. 2010;31:365-71.

14. Kim DH. Effect of Gamijakyakgamchobuja-tang on neuropathic pain in rats. first ed. Iksan:Wonkwang Uni- 
versity;2015

15. Kim HC, Kim YK.Genome-wide analysis on the effects of Salviae miltiorrhizae Radix in hyperlipidemic mice. Research Institute of Korean Medicine, Taejon University. 2013;21: 73-84.

16. Kim JK, Kim SH, Lee SM, Jeong HH, Park MY, Kim DW, et al. Study of Single-dose Toxicity of Aconitum Kusnezoffii Reichb. Pharmacopuncture in Rats. Jounal of Pharmacopuncture. 2012;15(3): 48-52.

17. Kim YH, Seo BI. A philological study on poisoning and side effects of Gastrodiae Rhizoma. The Journal of Jeahan Oriental Medical Academy. 2012;10:137-45.

18. Kwak JS, Baek SH. Cytotoxicity and antimicrobial effects of extracts from Salvia miltiorrhiza. Korean J. Pharmacogn. 2003;34:293-6.

19. Lee GM, Hwang WJ, Cho KY, Kim KY. The Effects of Aqua-acupuncture with Radix aconiti on the Contraction of Rat's Spinal Cord Injury. The Acupuncture.1999;16:283-96.

20. Lee HD, Song CH. Effect of Glycyrrhizae Radix Herbal acupuncture on PCA and Secretion of $\beta$ Hexosaminidase and Cytokines in RBL-2H3 Cells. Korean J. Acupunct.2011; 28:77-83.

21. Lee HG, Kim YS. Effects of Salvia Miltriorrhiza Radix on Neuronal Apoptosis following Intracerebral Hemorrhage of Rats. The Korea Journal of Herbology. 2012;27:89-94.

22. Lee JM, Hong SY, Oh MS. Effects of Jakyakkamchobuja-tang on Papain-induced osteoarthritis in mice. J. Korean Orient. Med. 2013;34:116-135.

23. Miao Q. New advance in identification of Chinese medicine tianma. China Medical Herald. 2011;8: 20-5.

24. Oh HI, Park HB, Ju MS, Jung SY, Oh MS. Comparative Study of Anti-oxidant and Anti-inflammatory Activities between Curcumae longae Radix and Curcumae longae Rhizoma. The Korea Journal of Herbology .2010;25:83-91.

25. Oh HS, Kim BW. Anti-inflammatory activity of the water extract of Polygala tenuifolia Willd. Korean J. Orient. Int. Med. 2013;34: 204-14.

26. Park HJ, Shim HS, Kim H, Kim KS, Lee H, Hahm DH, et al. Effects of Glycyrrhizae Radix on Repeated Restraint Stress-induced Neurochemical and Behavioral Responses. Korean J. Physiol. Pharmacol. 2010;14:371376.

27. Park HS, Sohn SH. Effect of Paeonia Radix Rubra aqua-acupuncture on Adjuvant Arthritis in rats. The Acupuncture. 2000;17:153-68.

28. Quiles JL, Mesa MD, Ramírez-Tortosa CL, Aguilera CM, Battino M, Gil A, et al. Curcuma longa extract supplementation reduces oxidative stress and attenuates aortic fatty streak development in rabbits, Arterioscler. Thromb. Vasc. Biol.2002; 22: 1225-1231.

29. Seo SC, Um MS, Park WW, Ko HI. Licorice-induced hypokalemia and myopathy. Kidney Res. Clin. Pract.2006; 25:651-656.

30. Shin DS, Kim YI, Lee H. Effect of Herbal-acupuncture with Chaenomelis Fructus Solution at the Joksamni of Mice with Collagen-induced Arthritis. The journal of Korean Acupuncture \& Moxibustion Medicine Society. 2007;24:127-43.
31. Shin IJ, Son SU, Park H, Kim Y, Park SH, Swanberg K, et al. Preclinical evidence of rapid-onset antidepressant-like effect in Radix Polygalae extract. PLOS ONE. 9.2014;doi: e88617. https://doi.org/10.1371/journal. pone.0088617.

32. Singhuber J, Zhu M, Prinz S, Kopp B. Aconitum in traditional Chinese medicine: a valuable drug or an unpredictable risk?. J of Ethnopharmacol. 2009;126:18-30.

33. Tong $\mathrm{P}$, Wu C, Wang X, Hu H, Jin H, Li C, et al. Development and assessment of a complete-detoxication strategy for fuzi (lateral root of Aconitum carmichaeli) and its application in rheumatoid arthritis therapy. J Ethnopharmacol. 2013;146: 562-71.

34. Yang SA, Lim NK, Lee IS. Effects of methanolic extract from Salvia miltiorrhiza bunge on in vitro antithrombotic and antioxidative activities. Korean J. Food SCI Technol. 2007; 39:83-7.

35. Yu JW. Study on the ingredient of Jakyakgamchotang. first ed. Iksan: Wonkwang University;2010. 